\title{
Hybride Modellierung deichbruchinduzierter Strömungen
}

Experimentelle Modellversuche und numerische Simulationen ergänzen sich gegenseitig in einem hybriden Untersuchungskonzept für deichbruchinduzierte Strömungen. Unter Berücksichtigung der deichbruchspezifischen Randbedingungen werden in Laborversuchen charakteristische Strömungsfeldgrößen mit hochgenauer Messtechnik detailliert aufgezeichnet. Die korrespondierenden numerischen Modellierungen werden auf Basis der zweidimensional tiefengemittelten Flachwassergleichungen sowie der Reynolds-gemittelten Navier-Stokes-Gleichungen durchgeführt.

\section{Einleitung}

\subsection{Motivation}

Binnendeiche sind Erdbauwerke des technischen Hochwasserschutzes, die Fließgewässer v. a. in dicht besiedelten Regionen beidseitig flankieren. Wenn diese Schutzeinrichtungen im Hochwasserfall versagen, wird durch die entstandene Bruchstelle eine Flutwelle in das vormals geschützte Deichhinterland ausgelöst. Wiederkehrende Überschwemmungen infolge Deichbrüchen belegen, dass ein Versagen der Schutzbauwerke nicht völlig auszuschließen ist und die resultierende Gefährdung deshalb erfasst werden muss. Moderne Hochwasserschutzkonzeptionen negieren deshalb eine absolute Sicherheit und bedienen sich stattdessen risikoorientierter Bemessungsphilosophien. Mit dem Verfahren RAPID (Risk Assessment: Probabilities, Inundation, Damage) wurde am Institut für Wasserbau und Wasserwirtschaft (IWW) der RWTH Aachen ein Risk-Assessment für Flussdeiche entwickelt [1]. Numerische Überflutungssimulationen einer Vielzahl hypothetischer Deichbruchszenarien können die hydrodynamischen Eingangsparameter, die Dauer des Ereignisses und die betroffenen Flächen zur Abschätzung des Risikos als Produkt aus Versagenswahrscheinlichkeit und den resultierenden Schäden bereitstellen [3].

Hinzu kommen die gesetzlichen Vorgaben auf Bundes- und EU-Ebene. Sowohl in der europäischen Richtlinie „über die Bewertung und das Management von Hochwasserrisiken“" (RL 2007/
60/EG) als auch im Wasserhaushaltsgesetzes (WHG) wird in einem mehrstufigen Ansatz die Bestimmung, Bewertung, Ausweisung und das Management des Hochwasserrisikos gefordert (z. B. $\$ \$ 73$ bis 75 WHG). In der RL 2007/60/EG wird dabei zur vorläufigen Bewertung des Risikos auf die „Wirksamkeit der bestehenden vom Menschen geschaffenen Hochwasserabwehrinfrastrukturen“ hingewiesen (Artikel 4 Absatz 2 Buchstabe d).

Die Deutsche Forschungsgemeinschaft (DFG) betont im Leitthema 5 ihrer Denkschrift „Wasser und Naturkatastrophen " [4], dass eine dringende Notwendigkeit zur angewandten und grundlegenden Forschung besteht: „Ob auf natürliche Weise oder durch den Bruch eines Deiches oder Dammes entstanden: Der Prozess der Ausbreitung in die Fläche und die an bestimmten Stellen wirkenden Strömungskräfte sind von besonderem Interesse. [...] Derzeit sind hydraulische Berechnungen für Flüsse mit entweder 1-D-Modellen (nach St. Venant) oder mit tiefengemittelten 2-D-Modellen üblich. Wenn der Fluss seine Ufer überströmt, sind diese Modelle jedoch nur in vereinfachter Form in der Lage, die Überflutungsmengen und Höhen zu berechnen. Hier liegt ein erhebliches Defizit in der exakten Erfassung dieser Vorgänge vor, wobei Labor- und vor allem Naturmessungen dringend erforderlich sind." Alle folgenden Ausführungen orientieren sich eng an Roger [2]. 


\subsection{Phänomenologie und Vorgehensweise}

\section{Klassifikation deichbruchinduzierter}

\section{Strömungen}

Strömungen nach einem Deichversagen weisen zu bestimmten Zeitpunkten Gemeinsamkeiten mit dammbruchinduzierten Strömungen bzw. seitlichen Entnahmen auf und können zwischen diesen artverwandten Grenzfällen eingeordnet werden (Bild 1). Im Unterschied zu Staudämmen sind Deiche nicht ständig eingestaut und flankieren die Fließgewässer als kilometerlange Linienbauwerke, so dass sich der kontinuierliche $\mathrm{Zu}$ fluss im Versagensfall in den Breschendurchfluss und den unterwasserseitigen Gerinneabfluss aufteilt. Diese Einwirkung ist permanent - das Flussbett wird nicht wie ein Reservoir vollständig entleert. Vielmehr stellt sich aufgrund der dauerhaften Zuströmung eine konstante Wassertiefe in der Deichbruchstelle ein, wodurch der Potentialunterschied und der sich ausbildende Breschendurchfluss für die gesamte Dauer eines Hochwasserereignisses erhalten bleiben.

Der Ablauf deichbruchinduzierter Strömungsvorgänge im realen Versagensfall ist großräumig, lang andauernd, hochgradig instationär, turbulent, transkritisch, ausgeprägt dreidimensional und mehrphasig. Außerdem handelt es sich um einen Freispiegelabfluss, der mit einer komplexen bewegten freien Wasseroberfläche über ursprünglich trockene, geometrisch komplizierte Sohlentopografien und bewachsene bzw. bebaute Gelände- strukturen abläuft. Aufgrund der phänomenologischen Unterschiede zwischen den resultierenden Strömungen infolge eines Damm- und Deichversagens lassen sich die umfangreichen Kenntnisse $\mathrm{zu}$ dammbruchinduzierten Strömungen nicht ohne Einschränkungen auf die vorliegende Problemstellung übertragen. Die zentrale Fragestellung zur Entsprechung des oben formulierten Bedarfs betrifft die Verlässlichkeit und damit die Aussagekraft der numerisch ermittelten Ergebnisgrößen auch im Hinblick auf deren weitere Verwendung und Interpretation.

\section{Hybride Modellierung}

Zur Lösung dieser Fragestellung werden unterschiedliche zwei- und dreidimensionale numerische sowie ein experimentelles Modellierungsverfahren in einem hybriden Ansatz aufeinander aufbauend und wechselseitig ergänzend realisiert. Hybrida stammt aus dem Lateinischen und bedeutet Mischling bzw. von zweierlei Herkunft. Besser bekannt ist die griechische Hybris als schuldhafte Tat wider der natürlichen Ordnung und als frevelhafter Übermut. Die Gemeinsamkeit ist, dass eine bestimmte Ordnung durch etwas Gemischtes, aus Verschiedenartigem zusammengesetztes überschritten wird. Die Besonderheit eines hybriden Ansatzes liegt darin, dass die zusammengebrachten Modelle für sich genommen zwar bereits eigenständige Lösungswege darstellen, durch deren Kombination aber neue Ergebnisse entste-

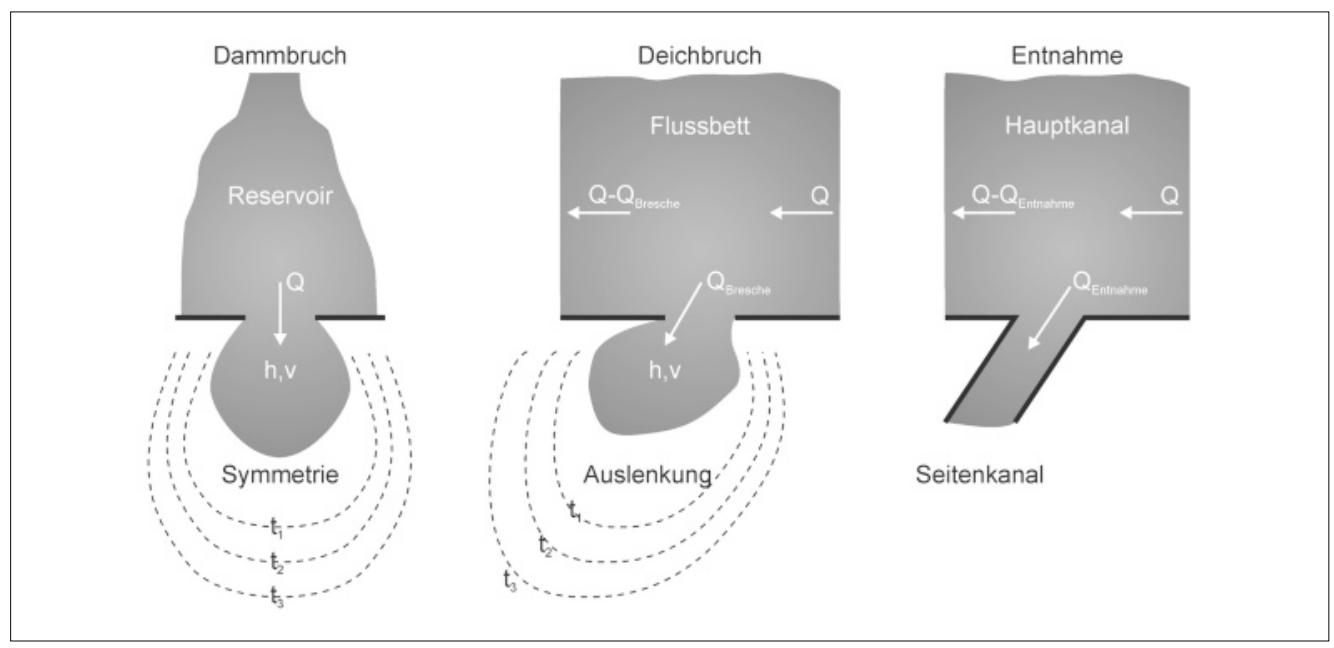

Bild 1: Klassifikation der artverwandten Strömungsbedingungen 
hen sollen und damit ein Mehrwert geschaffen werden kann (Synergieeffekt).

Der Charme einer hybriden Modellierung liegt nun darin, die Vorzüge beider Modellansätze zu vereinen und dabei die jeweiligen Schwächen zu kompensieren: Um die Natur im Modellversuch abzubilden, muss sie skaliert und idealisiert werden. Dies führt zu Abweichungen der im Labormaßstab beobachteten und vermessenen Vorgänge von den natürlichen Vorgängen. Um die Natur numerisch abzubilden, müssen vereinfachende Annahmen zur mathematisch-numerischen Beschreibung der Strömung getroffen werden.

Die Prognosegüte des numerischen Modells wird durch den Vergleich zu den Messungen im Modellversuch genau quantifizierbar, d. h. experimentelle Untersuchungen können der Entwicklung, Verifizierung und Validierung numerischer Berechnungsverfahren dienen. Skalierungseffekte und die modellbautechnischen Vereinfachungen haben dabei keinen Einfluss, wenn die experimentellen Strömungskonfigurationen auch im Labormaßstab numerisch simuliert werden. Gleichzeitig ergänzt das numerische Modell den Modellversuch, da in der Numerik alle szenarienspezifischen Geometrie-,
Bewuchs- oder Materialparameter sowie die Anfangs- und Randbedingungen leicht systematisch variiert werden können (Variantenstudien, Sensitivitätsanalysen).

\section{Experimentelle Modellierung}

\subsection{Aufbau der strömungsphysikalischen Modellversuche}

Für das Nahfeld einer Bresche wurden strömungsphysikalische Modellversuche so konzipiert, dass die speziellen Randbedingungen des deichbruchinduzierten Strömungstyps berücksichtigt werden können [5]. Die Versuchseinrichtung besteht aus einem gemauerten und verputzten Kanal (Länge ca. 8,0 m, Breite 1,0 m) mit rechteckigem Querschnitt ohne Sohlenneigung, der über eine pneumatisch betriebene Klappe seitlich an eine horizontale Glasfläche (Länge 4,0 m, Breite 3,5 m) angeschlossen ist (Bild 2). Mit diesem Modellaufbau kann ein Versagen von Schutzeinrichtungen im Hochwasserfall kleinmaßstäblich abstrahiert und idealisiert abgebildet werden: Der Kanal repräsentiert einen Hochwasser führenden Fluss, der seitlich von Schutzstruk-

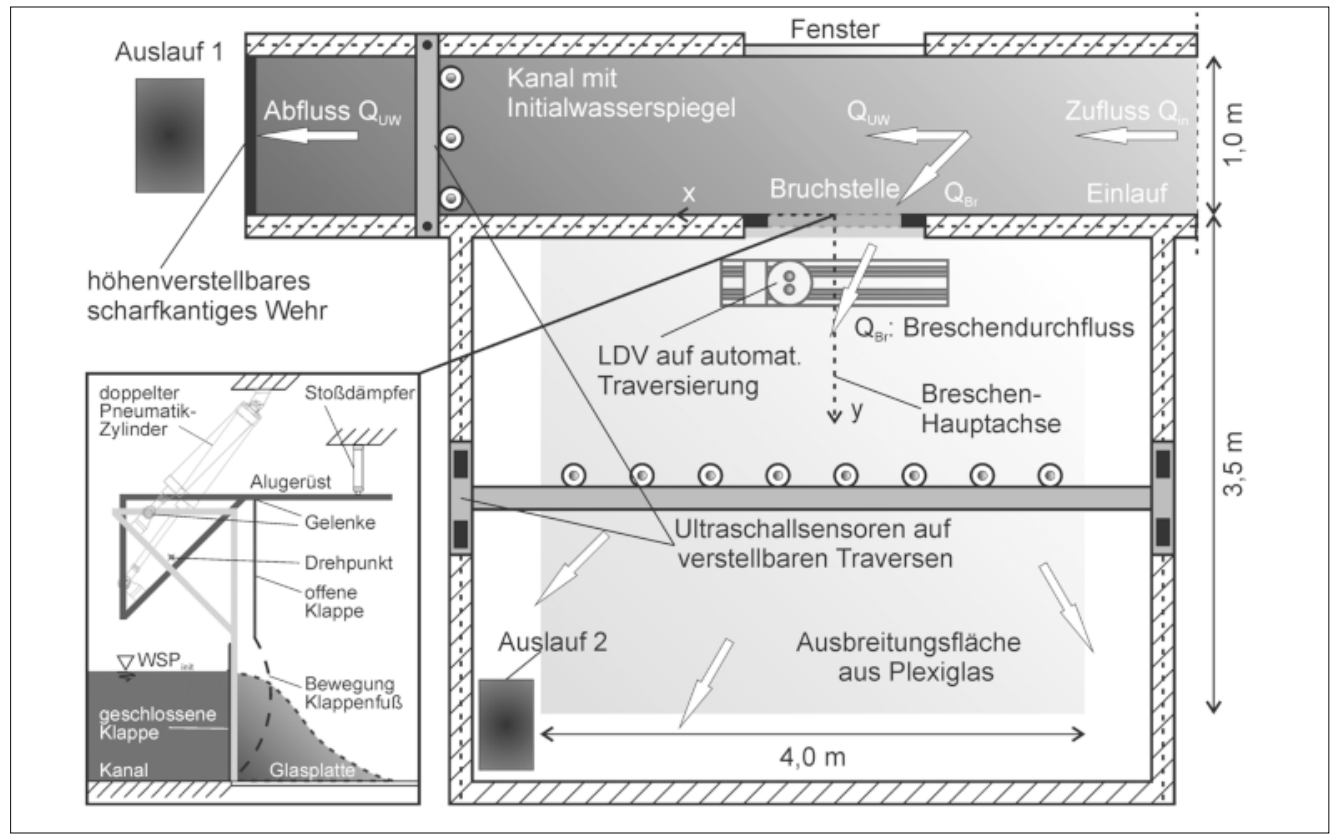

Bild 2: Versuchsaufbau des Deichbruchmodells 
turen flankiert wird. Die gläserne Ausbreitungsfläche stellt bei geschlossener Klappe das geschützte Hinterland da, welches im Versagensfall (Klappenöffnung) schlagartig überströmt wird. Im Gegensatz zu Versuchsrinnen, die eine flächige Flutwellenausbreitung verhindern, kann hier eine 3-D-Flutwellenausbreitung modelliert werden, da die Ablauffläche an drei Seiten nicht begrenzt wird und das Wasser an diesen Rändern reflexionsfrei überfallen kann. Die ebene Glaskonstruktion ermöglicht außerdem Messungen von unterhalb der Platte und minimiert dabei gleichzeitig die Einflüsse, die durch die Sohlenrauheit und das Sohlengefälle bedingt werden. Der Anfangswasserspiegel im Kanal hInit (0,3 bis 0,5 m), der Volumenstrom am Modelleinlauf QEin $\left(0,1\right.$ bis $\left.0,3 \mathrm{~m}^{3} / \mathrm{s}\right)$ und die Klappen- bzw. Breschenbreite $\mathrm{bBr}(0,3$ bis $0,7 \mathrm{~m})$ werden systematisch variiert.

\subsection{Messtechnik}

Am Modelleinlauf wird der Zufluss per Ultraschall nach dem Laufzeitdifferenzenverfahren gemessen. Der Abfluss über das kalibrierte scharfkantige Auslaufwehr am Kanalende wird mittelbar über Wasserstandmessungen bestimmt. Der stationäre Breschendurchfluss $\mathrm{Q}_{\mathrm{Br}}$ wird nicht direkt gemessen, sondern ergibt sich dann aus der Differenz von Zufluss am Modelleinlauf QEin und dem Abfluss QAus über das Auslaufwehr.

Die Wasserspiegellagen wurden über Ultraschallsensoren (USS) durch das Impuls-EchoVerfahren flächendeckend in einem Raster mit $\Delta \mathrm{x}=\Delta \mathrm{y}=0,1$ bis $0,2 \mathrm{~m}$ erfasst. Bis zu 8 USS gleichzeitig wurden nebeneinander an horizontal verstellbaren Traversen montiert, um die Fließtiefen an beliebiger Stelle auf der Glasplatte oder im Kanal messen zu können.

Die horizontalen Geschwindigkeitskomponenten werden nach dem Prinzip der Laser-DopplerVelocimetry (LDV) aufgenommen. Das LDVSystem wurde auf einem Laufschienensystem unterhalb der Glasplatte installiert, so dass an drei Querschnitten im Abstand von 0,25, 0,30 und $0,35 \mathrm{~m}$ von der Bresche eine automatisierte $\mathrm{Ab}$ tastung der horizontalen Geschwindigkeitskomponenten in einem Raster mit $\Delta \mathrm{x}=0,05$ und $\Delta \mathrm{z}=0,0133 \mathrm{~m}$ möglich war.

\section{Numerische Modellierung}

\subsection{Gesamtprozess einer hydronumerischen Modellierung}

Auf die mathematisch-physikalische Modellierung turbulenter Freispiegelströmungen soll hier nur insoweit eingegangen werden, dass für die 3-DBerechnungen die Reynolds-gemittelten NavierStokes-Gleichungen in Verbindung mit einem k- $\varepsilon$-Zweigleichungsmodell zur Turbulenzmodellierung und einer Volume-of-Fluid-Methode (VOF-Methode) zur Erfassung der freien Wasseroberfläche verwendet werden. Für die 2-D-Simulationen kommt die konservative Form der tiefengemittelten 2-D-Flachwassergleichungen zum Einsatz, wobei unterschiedliche Ansätze zur Berücksichtigung der Effekte aus Turbulenz, Bodenoder Wandrauheit genutzt werden. Eine hydronumerische Modellierung besteht nicht nur aus der Beschaffung und Anwendung eines Simulationsprogramms, sondern beinhaltet eine Vielzahl von Teilschritten (Bild 3). Diese sind so komplex, dass der gesamte Prozess arbeitsaufwendig, zeit- und kostenintensiv mit äußerster Sorgfalt durchgeführt werden muss, wenn eine qualitativ hochwertige Modellierung erreicht werden will [6].

\subsection{Zur Programmauswahl}

Aus dem Gesamtprozess der numerischen Modellierung wird hier der Teilschritt Programmauswahl herausgegriffen. DGFlow wurde von Schwanenberg [7] am IWW der RWTH Aachen entwickelt und mit analytisch lösbaren 1-D-Testproblemen besonders im Hinblick auf transkritische Strömungen umfassend verifiziert. Die Validierung wurde auf Grundlage einer überkritischen Strömung in einer sich verengenden Schussrinne und anhand der realen Flutwellenausbreitung des Dammbruchs von Malpasset durchgeführt [8].

WOLF 2D wird in der Abteilung „Hydraulics in environmental and civil engineering " (HECE) der Universität Lüttich stetig weiter entwickelt. Im Rahmen der engen Kooperation zwischen HECE und IWW konnte die Anwendbarkeit auf deichbruchinduzierte Strömungen getestet werden [9]. Das kommerzielle Programm STAR-CD von CDadapco ist das Nachfolgemodell des Programmsystems Comet, mit dem am IWW einige Erfah- 


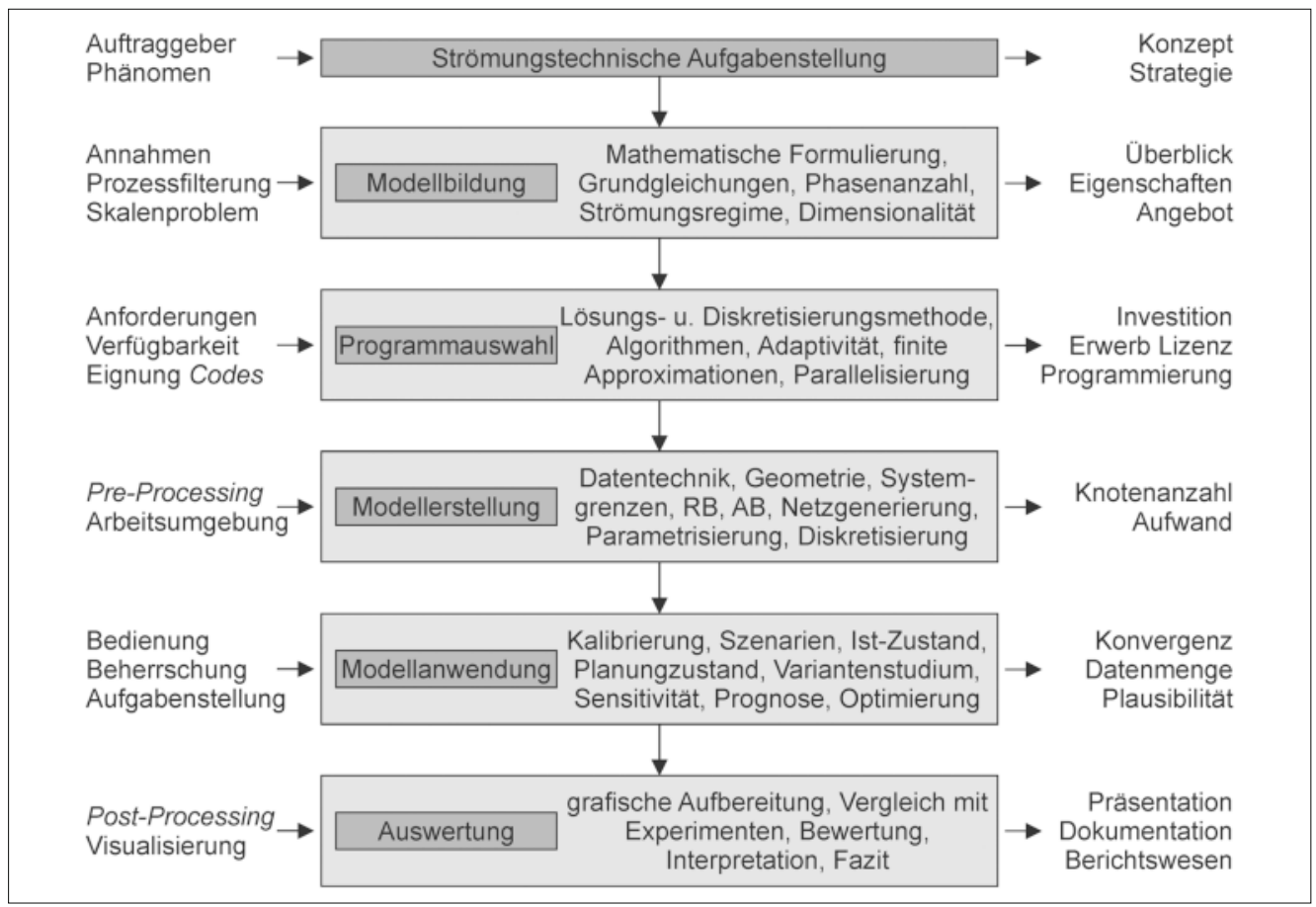

Bild 3: Prozessschritte einer hydronumerischen Modellierung

rungswerte in Bezug auf die 3-D-Simulation wasserbaurelevanter Strömungsprobleme bestehen [10].

\section{Auswertung}

\subsection{Bestimmung des stationären Breschendurchflusses}

Obwohl die Durchflussaufteilung in den Breschendurchfluss und den unterwasserseitigen Gerinneabfluss in allen Modellrechnungen qualitativ gut erfasst wird, unterschätzen die Flachwassermodelle DGFlow und WOLF 2D den Abfluss, der sich über die Ausbreitungsfläche ergießt, um $4 \%$ bis $11 \%$ (bezogen auf die Messwerte). Die 3-D-Resultate aus STAR-CD zeigen - hypothesenkonform - eine deutlich bessere Übereinstimmung mit den Messwerten für die dargestellten Konfigurationen (Bild 4). Trotzdem werden auch mit STAR-CD die experimentellen Werte nicht exakt berechnet. Bemerkenswert ist die Tatsache, dass die beiden Flachwassermodelle für sich genommen sehr gut zusammenpassen.

\subsection{Flachwasserapproximationen}

Um quantitative Aussagen treffen zu können, wurden die numerisch ermittelten Fließtiefen mit den experimentell erfassten Werten verglichen. Es zeigte sich, dass die beiden Flachwassermodelle trotz ihrer komplett unterschiedlichen numerischen Methoden die Fließtiefen in sehr guter Übereinstimmung untereinander berechnen [9]. Mit WOLF 2D wurden Berechnungen daraufhin mit einem algebraischen Turbulenzmodell und einem Zweigleichungsmodell durchgeführt (Bild 5). Außerdem wurden Szenarien mit einem verlängerten Einlaufkanal sowie mit Wand- und Sohlenrauheitsparametrisierungen simuliert. Beide Modelle sind dabei in der Lage, das deichbruchinduzierte Strömungsfeld im Wesentlichen zu reproduzieren. Dies schließt die qualitative Erfassung der Durchflussaufteilung für die verschiedenen experimentellen Konfigurationen der Modellversuche mit ein. Die Strömungsvorgänge wurden von einem Initialzustand mit geschlossener Klappe aus gestartet und in einem oszillationsfreien Berechnungslauf simuliert, womit die generelle Anwendbarkeit und Stabilität der Pro- 

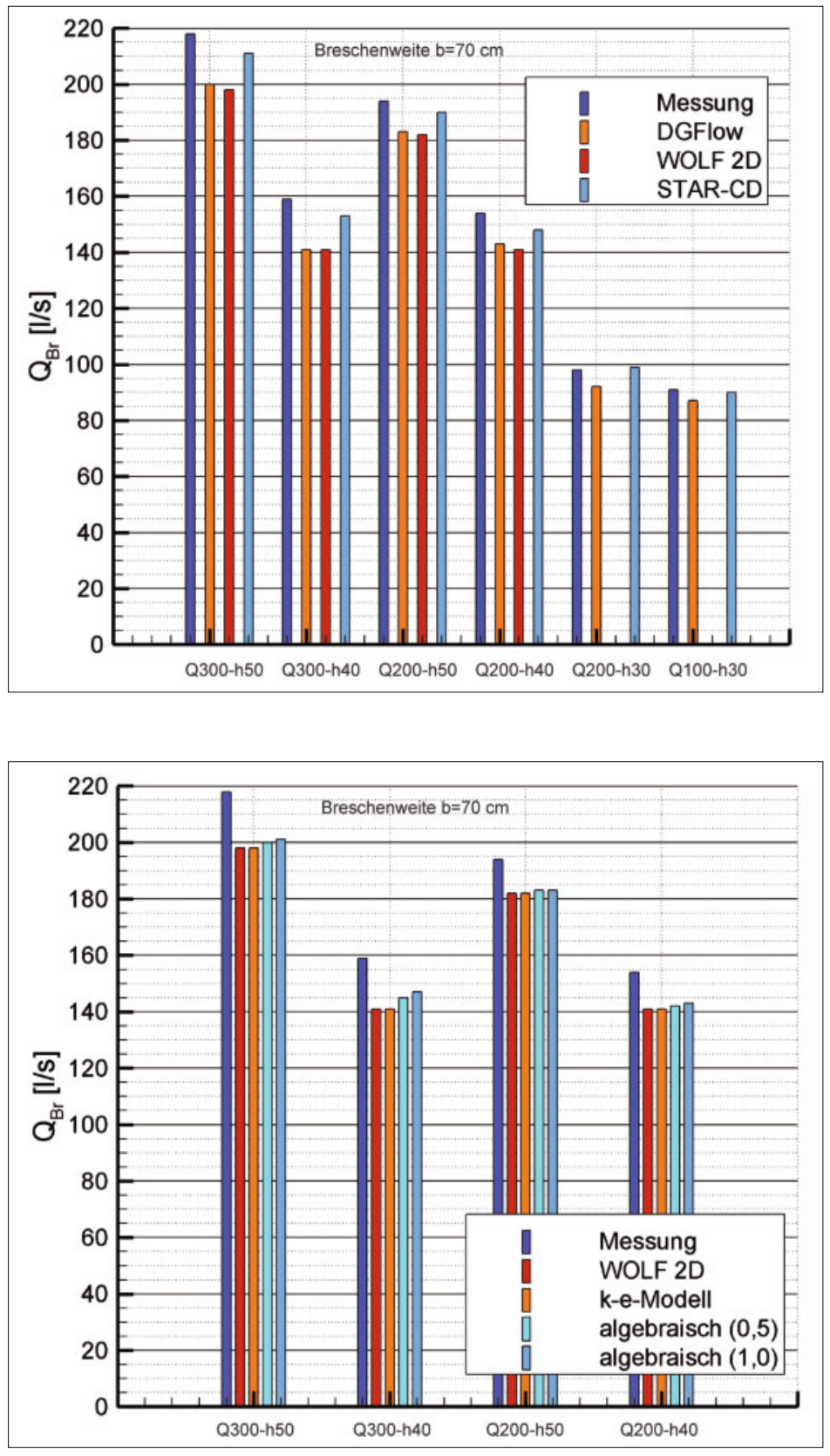

Bild 4: Gemessene und berechnete Breschendurchflüsse $Q_{B r}$ bei einer Breschenweite von $70 \mathrm{~cm}$
Bild 5: Gemessene und mit unterschiedlichen Turbulenzmodellen berechnete Breschendurchflüsse $\mathrm{Q}_{\mathrm{Br}}$ gramme für den deichbruchinduzierten Strömungstyp bestätigt wird. Die Simulation der dynamischen Flutwellenausbreitung bis hin zum stationären Endzustand umfasste den Kanal und die Glasfläche in einer umhüllenden Berechnungsdomäne.
Die Abweichungen von den Messungen mit einer Unterschätzung des Breschendurchflusses von $4 \%$ bis $11 \%$ wurden analysiert, indem die Vereinfachungen des mathematischen Modells und der numerischen Ansätze abgeprüft wurden. Die untersuchten Testfälle offenbarten eine 
sehr geringe Sensitivität sowohl gegenüber der Turbulenzmodellierung als auch hinsichtlich der Parametrisierung Boden- und Wandrauheit [9].

\subsection{2-D-/3-D-Vergleich}

Bild 6 zeigt den Wasserspiegellagenverlauf entlang einer Kanalachse mit y = -0,2 m. Das Wasser fließt von links nach rechts (Blickrichtung Schnitt A-A). Nach Klappenöffnung bildet sich ein stationärer Wechselsprung im Breschennahfeld aus, der durch die 3-D-Numerik signifikant besser erfasst wird. Der Wasserspiegel wird in den 2-D-Berechnungen stromab der Bresche überschätzt. Dadurch erklären sich auch die zu hohen unterwasserseitigen Gerinneabflüsse (größere Wehrüberfallhöhen am Ende des Kanals), die wiederum in den zu geringen Breschendurchflüssen resultieren. Die Aufteilung der Durchflüsse und die sich dadurch ergebenen Strömungsverhältnisse im wasserseitigen Breschennahfeld werden demnach mit beiden Flachwassermodellen nicht exakt abgebildet.

\subsection{Geschwindigkeiten}

Bild 7 zeigt eine synoptische Darstellung der gemessenen sowie auf unterschiedlichem Wege berechneten Fließgeschwindigkeiten. Im Hintergrund sind die gemessenen horizontalen Fließgeschwindigkeiten über die Fließtiefe als Vektoren in den unterschiedlichen Höhen abgebildet. Die Länge der Vektoren entspricht dem absoluten Betrag der Horizontalgeschwindigkeit. Durch die Farbgebung wird die Ausrichtung gekennzeichnet. Eine Gelbfärbung entspricht dabei einer Geschwindigkeitsrichtung exakt senkrecht zur Klappe, d. h. in Richtung Breschenhauptachse. Grünlich bis bläuliche Einfärbungen stehen für eine anwachsende Auslenkung in Richtung der positiven $\mathrm{x}$-Achse entsprechend der Kanalhauptströmung, während rote und purpurne Farbtöne eine negative $\mathrm{x}$-Richtung anzeigen. Im Vordergrund sind die berechneten Vektoren in den unterschiedlichen Höhenlagen eingezeichnet. Die Vektoren aus den 3-D-Simulationen (STAR-CD) unterliegen der gleichen Farbgebung für die Auslenkung. Durch die berechnete vertikale Geschwindigkeitskomponente können die Vektoren zusätzlich Ausdehnungen

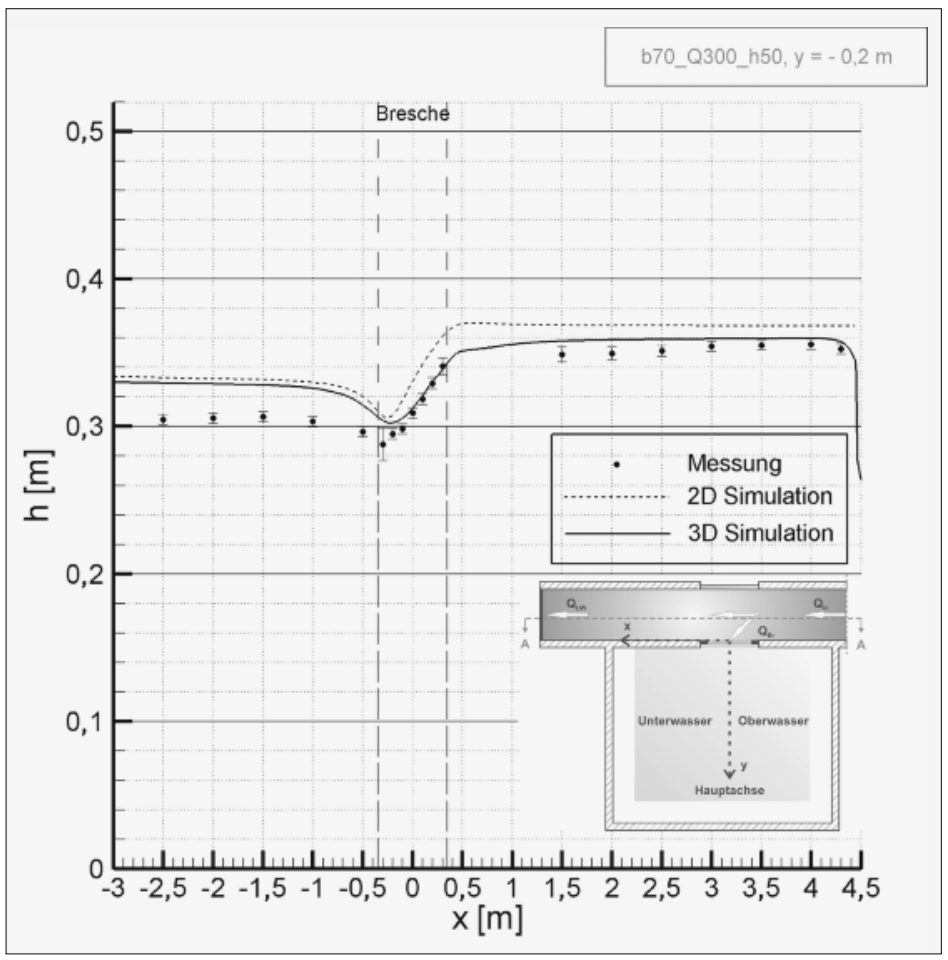

Bild 6: Wasserspiegellagenvergleich: 2-D-/3-DMessung für den Schnitt y $=-0,2 \mathrm{~m}$ 


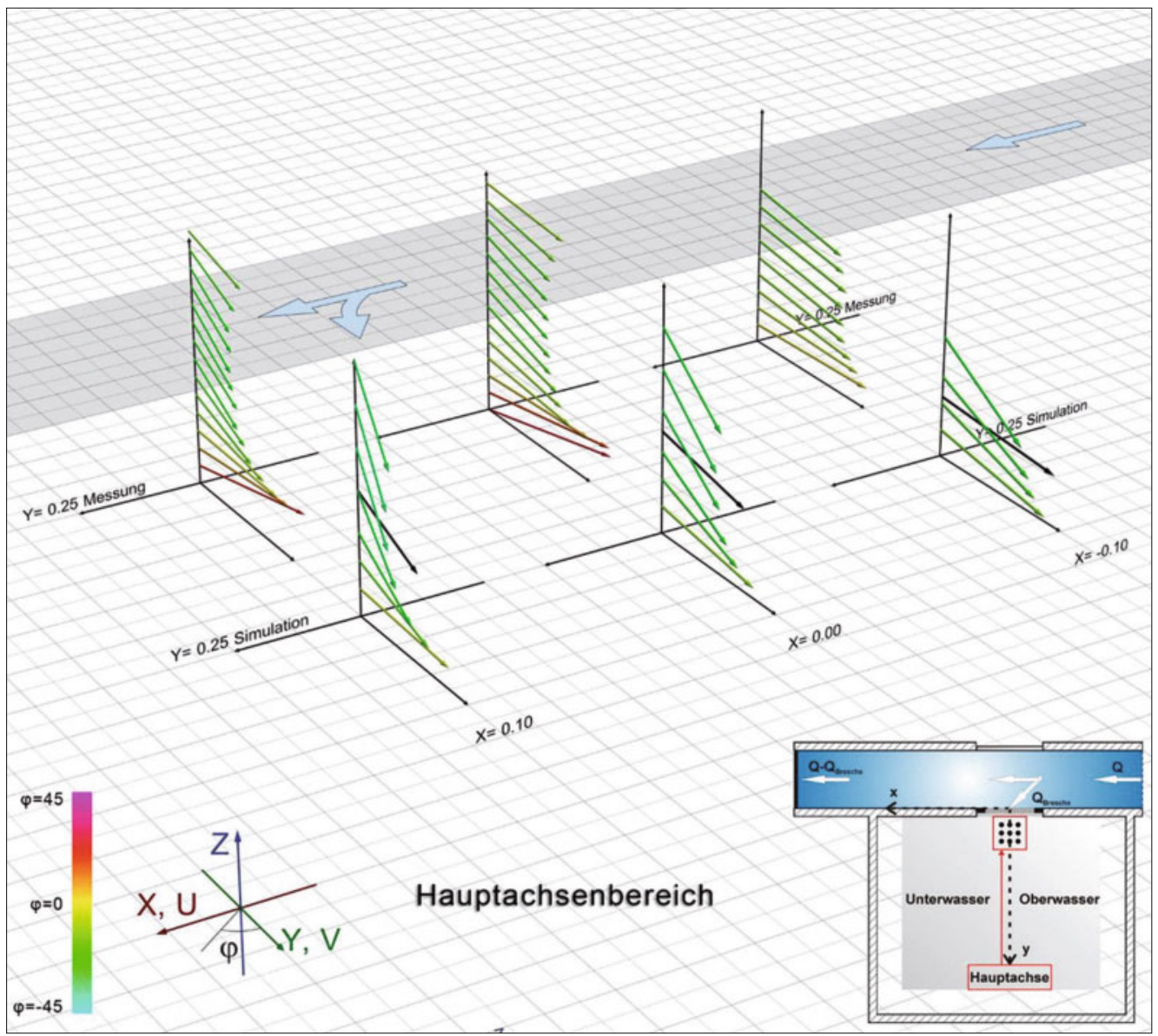

Bild 7: Vergleich von Geschwindigkeitsvektoren: 2-D-/3-D-Messung (Schrägansicht)

entlang der z-Achse aufweisen. Die Pfeillänge repräsentiert den Betrag der räumlichen Resultierenden. In das simulierte Tiefenprofil ist auf halber Fließtiefe der aus der Flachwassernumerik (DGFlow) tiefengemittelte Geschwindigkeitsvektor in schwarz mit seiner betragsmäßigen Länge dargestellt.

In der Schrägansicht (Bild 7) lässt sich für die Messungen die Verwindung der Tiefenprofile gut erkennen. Alle 3-D-Berechnungen überschätzen den $\mathrm{x}$-Impuls und die Tiefenprofile sind teilweise noch mehr in die positive $\mathrm{x}$-Richtung ausgedreht. Qualitativ werden die oberen Bereiche der Geschwindigkeitsprofile über die Fließtiefe auch in ihren Verläufen besser erfasst. Jedoch ist die berechnete Verwindung nicht so ausgeprägt, dass die sohlennahen Vektoren in negative x-Richtung weisen würden. Alle berechneten Fließvektoren liegen im 1. Quadranten, wobei sich die sohlennahen 3-D-Vektoren der Breschenhauptachse annähern. Der tiefengemittelte Geschwindigkeitsvektor aus den Flachwassersimulationen repräsentiert in Richtung und mit Betrag die 3-DBerechnungen recht gut, wobei er die 3-D-Effekte, wie die Verwindung und den Vorzeichenwechsel der u-Komponente, bei den Messungen nicht abzubilden vermag.

Bei einer anderen Konfiguration wird aus der Vogelperspektive die Streuung in positive bzw. negative $\mathrm{x}$ - Richtung durch die 3-D-Simulationen gut erfasst und der Vorzeichenwechsel im Tiefenprofil bei $\mathrm{x}=0,0 \mathrm{~m}$ wird mit STAR-CD abgebildet (Bild 8). Außerdem stimmen die Orientierung in negative (bei $\mathrm{x}=-0,1 \mathrm{~m}$ ) und in 


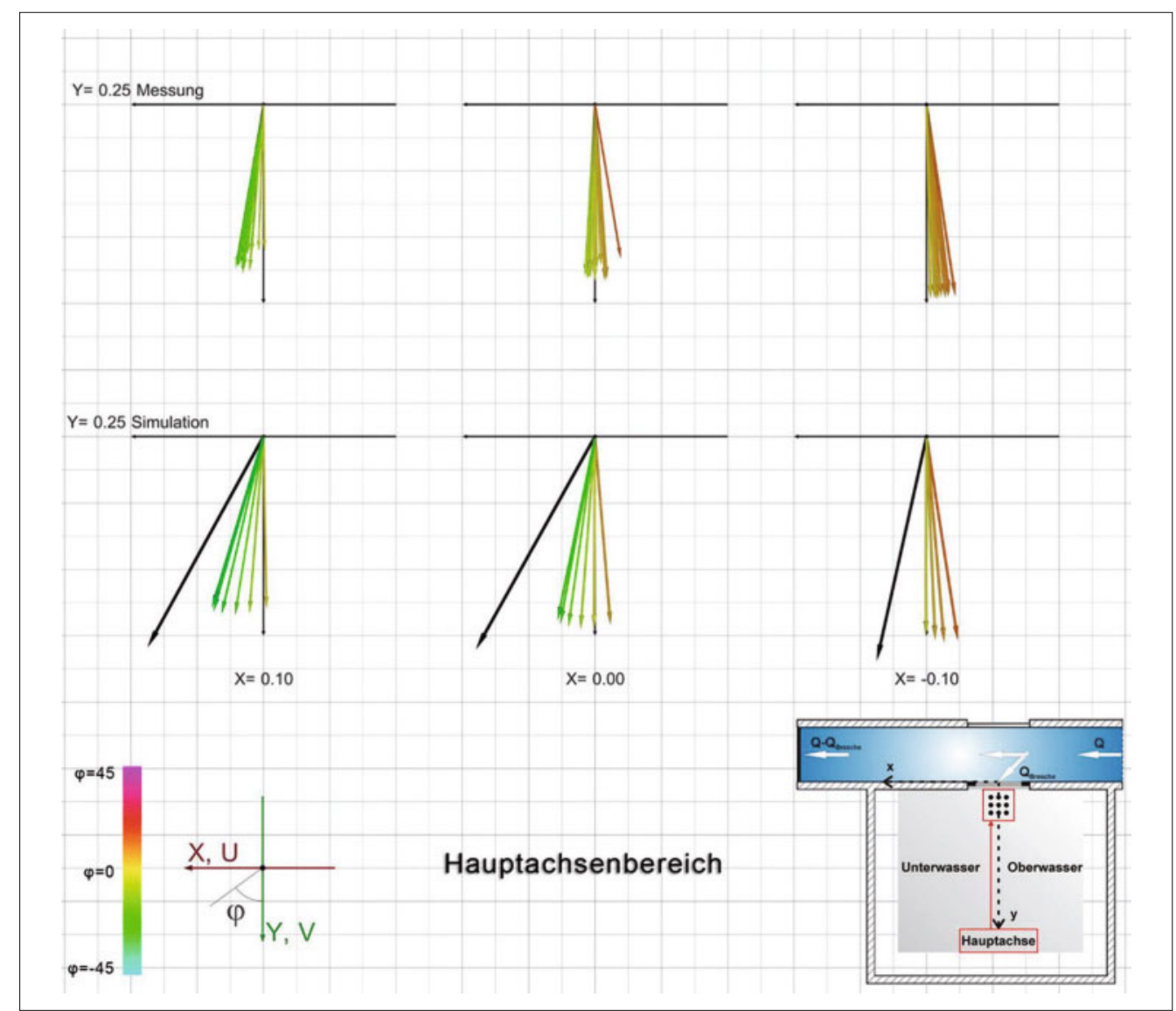

Bild 8: Vergleich von Geschwindigkeitsvektoren: 2-D-/3-D-Messung (Draufsicht)

positive (bei $\mathrm{x}=0,1 \mathrm{~m}$ ) $\mathrm{x}$-Richtung sowie die Beträge sehr gut überein. In diesem Fall versagen die Flachwasserberechnungen fast vollständig, da sowohl Richtung als auch Betrag der simulierten Geschwindigkeiten signifikant abweichen.

\section{Resümee}

\subsection{Schlussfolgerungen Modellversuche}

Im Zuge der experimentellen Arbeiten des hybriden Ansatzes wird die Erfassung der Durchflüsse durch eine erweiterte Parametrisierung der Wehrcharakteristika am Kanalauslauf erheblich verbessert. Die resultierenden kalibrierten Wehrüberfallformeln werden als untere Randbedingung bei den Flachwassersimulationen in die Codes von DGFlow und WOLF 2D implementiert. STAR-
CD kommt ohne den Ansatz einer Wehrrandbedingung aus, da die Auslaufrandbedingung erst nach dem Fließwechsel positioniert wird (hydraulische Entkoppelung) und der Wehrkörper ausschließlich über die Netzgeometrie ausdiskretisiert wird.

Die Wasserspiegellagen werden flächendeckend für den stationären Zustand mit Ultraschallsensoren in einem dichten Raster auf der Glasfläche und im Kanal aufgemessen. Die Ausbreitungsfläche besteht aus Glas, wodurch Rauheitseffekte minimiert werden und eine messtechnische Zugänglichkeit von unterhalb der Glasplatte besteht, so dass eine automatisierte Detektion von Tiefenprofilen der Horizontalgeschwindigkeiten innerhalb des Wellenkörpers im luftseitigen Breschennahfeld realisiert werden kann. Daraus resultiert eine vollkommen neue Datenmenge aus Strömungsfeldgrößen. 


\subsection{Schlussfolgerungen Numerik}

Der stationäre Breschendurchfluss wird bei der hier angewandten Numerik in allen Fällen unterschätzt. Die Abflüsse durch die Bruchstelle werden von den Flachwassermodellen DGFlow und WOLF 2D bezogen auf die Messwerte um $4 \%$ bis $11 \%$ zu niedrig berechnet. Die Simulationsergebnisse mitSTARCD erreichen einen deutlich höheren Deckungsgrad mit den Messwerten und berechnen für drei Konfigurationen sogar sehr genaue Werte.

Bei einem 2-D-internen Vergleich ist es bemerkenswert, dass beide 2-D-Codes untereinander Ergebnisse mit sehr guter Übereinstimmung berechnen. Dies gilt nicht nur für den Breschendurchfluss als integralen Summenparameter, sondern auch für ausgezeichnete Wasserspiegellagen. Es handelt sich bei DGFlow und WOLF 2D um grundsätzlich verschiedene numerische Verfahren, die mit unterschiedlichen Diskretisierungsmethoden, Gitterentwürfen und Lösungsalgorithmen identische Lösungswerte ermitteln. Mit WOLF 2D können unterschiedliche Turbulenzmodelle und Rauheitsansätze für Seitenwände modular und räumlich verteilt angesetzt werden. Eine Variation der Bodenrauheitsbeiwerte wird mit DGFlow vollzogen. Die Simulationsläufe manifestieren eine geringe Sensitivität der Durchflussaufteilung in Breschendurchfluss und unterwasserseitigen Gerinneabfluss bezogen auf Turbulenzeffekte sowie Sohlen- und Wandrauheiten.

Der 2-D-/3-D-Vergleich der Wasserspiegellageprofile an ausgezeichneten Schnitten durch das Berechnungsgebiet fällt eindeutig zu Gunsten der 3-D-Numerik aus. Der Verlauf und die Absolutwerte der Fließtiefen im Kanal und in Breschennähe werden mit STAR-CD signifikant genauer abgebildet.

Das komplexe Geschwindigkeitsfeld im Nahbereich der Bruchstelle wird durch die 3-D-Numerik im Prinzip wiedergegeben. Dies beinhaltet die Beträge der Geschwindigkeiten und die Orientierung der Vektoren. Auch die in sich verdrehten Tiefenprofile werden erfasst, jedoch nicht immer an der richtigen Position. Durch Ausdehnung des Auswertebereichs (STAR-CD) im Vergleich zum Messbereich kann gezeigt werden, dass die angesprochenen Phänomene Berücksichtigung finden, wenn auch weiter entfernt von der Bresche. Der tiefengemittelte Geschwindigkeitsvektor kann die 3-D-Effekte naturgemäß gar nicht abbil- den. Jedoch zeigt sich vereinzelt an homogeneren Tiefenprofilen, dass er z. T. erheblich von der erwarteten Tiefenmittelung der gemessenen bzw. dreidimensional simulierten Verhältnisse abweicht.

\subsection{Fazit und Ausblick}

Da der stationäre Breschendurchfluss ein entscheidender Parameter besonders für die langfristige Überflutungssimulationen ist, stellt er eine der wichtigsten numerischen Zielgrößen bei der Bewertung der Modellgenauigkeit dar. Er inkorporiert als Integralparameter viele Strömungsprozesse an der Bresche, ist verhältnismäßig einfach zu bestimmen und erlaubt eine anschauliche Auswertung. Außerdem werden die relevanten hydrodynamischen Überflutungsgrößen im Deichhinterland von ihm abgeleitet. Aus diesem Blickwinkel kann man zur korrekteren Bestimmung der Abflussmengen durch eine Deichbruchstelle nur eine vollständige 3-D-Simulation für den breschennahen Fließgewässerabschnitt empfehlen.

Viele praxisrelevante wasserbauliche Anwendungen haben gezeigt, dass es vorteilhaft sein kann, mit vielen Gitterpunkten und einfachen Methoden zu arbeiten, anstatt mit höchst anspruchsvollen Methoden zu arbeiten und dabei die hohe Genauigkeit mit weniger Netzpunkten erreichen $\mathrm{zu}$ wollen. Die 2-D-Rastermethoden auf Storage-Cell-Basis folgen dieser Philosophie. Das „Wunschmodell“ zur Betrachtung von statischen Schäden, zur Ausweisung überfluteter Flächen und zur Bereitstellung der hydrodynamischen Eingangsparameter für ein Risk Assessment wäre ein gekoppeltes 2-D-/3-D-Modell: Der Fließgewässerabschnitt und ein sehr kleiner Teil des Deichhinterlandes im Bereich der Bruchstelle werden dabei dreidimensional simuliert. Auf diese Weise wird der Breschendurchfluss bestmöglich ermittelt und kann für ein vereinfachtes rasterbasiertes Modell für das Überschwemmungsgebiet als obere Randbedingung angesetzt werden. Der Modellgebietsrand des 3-D-Modells muss im Hinterland nach dem Fließwechsel verortet sein, so dass eine hydraulische Entkopplung vorliegt. Das Storage-Cell-Modell beginnt ab dem Breschenquerschnitt. Beide Teilmodelle überlappen sich, so dass die Herausforderung in der Synchronisierung des Durchflusses im Breschenquerschnitt liegt. 
Für risikoorientierte Prognosesimulationen möglicher zukünftiger Szenarien, probabilistische Variantenstudien oder Erweiterungen in der mathematisch-physikalischen Modellierung ist eine Effizienzsteigerung bei der numerischen Berechnung zwingend erforderlich. Neben der gekoppelten Modellierung bieten sich parallele Simulationen auf mehreren Prozessoren an. Für das Flachwassermodell DGFlow sind bereits erste Schritte unternommen worden, indem ein Steigerung der Rechenleistung durch die Parallelisierung für Grafikprozessoren (GPU) mittels der Compute Unified Device Architecture (CUDA) erreicht wird [11].

\section{Danksagung}

Die vorliegende Publikation basiert in wesentlichen Teilen auf der Dissertation des Erstautors [2]. Besonderer Dank gilt Herrn Univ.-Prof. Dr.-Ing. Jürgen Köngeter, Herrn Univ.-Prof. Dr. rer. nat. Sebastian Noelle und Herrn Univ.-Prof. Dr.-Ing. Holger Schüttrumpf für die wissenschaftliche und fachliche Betreuung dieser Arbeit. Der Erstautor dankt weiterhin der DFG für die finanzielle Unterstützung im Rahmen des Forschungsvorhabens mit dem Förderkennzeichen KO 1573/15-2 [12]. Der Zweitautor dankt dem „Fonds de la Recherche Scientifique (Communauté francaise de Belgique) - F.R.S-FNRS“ sowie der Universität Lüttich (ARD and Duesberg Foundation) für die Förderung der Kooperation zwischen HECE und IWW.

\author{
Autoren \\ Dr.-Ing. Sebastian Roger \\ RWE Innogy GmbH \\ Gildehofstraße 1 \\ 45127 Essen \\ sebastian.roger@rwe.com
}

\section{Prof. Benjamin Dewals}

Hydraulics in Environmental and Civil Engineering (HECE)

Département ArGEnCo - Secteur MS ${ }^{2} \mathrm{~F}$

Université de Liège

Chemin des Chevreuils 1

4000 - Liège, Belgien

B.Dewals@ulg.ac.be

\section{Univ.-Prof. Dr.-Ing. Holger Schüttrumpf}

Lehrstuhl und Institut für Wasserbau und

Wasserwirtschaft der RWTH Aachen

Mies-van-der-Rohe-Straße 1

52056 Aachen

schuettrumpf@iww.rwth-aachen.de
Literatur

[1] Kutschera, G.; Bachmann, D.; Huber, N. P.; Niemeyer, M.; Köngeter, J.: RAPID - Ein Risk-Assessment-Verfahren für den technischen Hochwasserschutz. In: WasserWirtschaft 98 (2008), Heft 1-2, S. 43-48.

[2] Roger, S.: Hybride Modellierung deichbruchinduzierter Strömungen für ein idealisiertes Breschennahfeld an Fließgewässern. Dissertation am Lehrstuhl und Instituts, für Wasserbau und Wasserwirtschaft der RWTH Aachen, 2012.

[3] Niemeyer, M.; Huber, N. P;; Briechle, S.; Köngeter, J.: Simulation damm- und deichbruchinduzierter Flutwellen. In: Österreichische Wasser- und Abfallwirtschaft 57 (2005), Heft 1-2, S. 26-32.

[4] Deutsche Forschungsgemeinschaft (Hrsg.): Wasserforschung im Spannungsfeld zwischen Gegenwartsbewältigung und Zukunftssicherung. Denkschrift der Senatskommission für Wasserforschung der DFG, 2003.

[5] Briechle, S.: Die flächenhafte Ausbreitung der Flutwelle nach Versagen von Hochwasserschutzeinrichtungen an Fließgewässern. In: Mitteilungen des Lehrstuhl und Instituts für Wasserbau und Wasserwirtschaft der RWTH Aachen (2007), Nr. 149.

[6] Forkel, C.: Numerische Modelle für die Wasserbaupraxis: Grundlagen, Anwendungen, Qualitätsaspekte. In: Mitteilungen des Lehrstuhl und Instituts für Wasserbau und Wasserwirtschaft der RWTH Aachen (2004), Nr. 130.

[7] Schwanenberg, D.: Die Runge-Kutta-DiscontinuousGalerkin-Methode zur Lösung konvektionsdominierter tiefengemittelter Flachwasserprobleme. In: Mitteilungen des Lehrstuhl und Instituts für Wasserbau und Wasserwirtschaft der RWTH Aachen (2004), Nr. 135.

[8] Schwanenberg, D.; Harms, M.: Discontinuous Galerkin finite-element method for transcritical two-dimensional shallow water flows. In: Journal of Hydraulic Engineering 130 (2004), No. 5, pp. 412-421.

[9] Roger, S.; Dewals, B. J.; Erpicum, S.; Schwanenberg, D.; Schüttrumpf, H.; Köngeter, J.; Pirotton, M.: Experimental and numerical investigations of dike-break induced flows. In: Journal of Hydraulic Research 47 (2009), No. 3, pp. 349-359.

[10] Roger, S.: Numerical Approaches to Simulate Two-Phase Flows. In: Proceedings of XXXI IAHR Congress, Seoul, Korea, 2005, S. 6 453-6 464.

[11] Schwanenberg, D.; Horsten, S.; Roger, S.: Discontinuous Galerkin Shallow Water Solver on Cuda Architectures. In: Proceedings of the 9th International Conference on Hydroinformatics 2010, Tianjin, 2010, Vol. 2, S. 944-951.

[12] Roger, S.; Köngeter, J.; Schüttrumpf, H.: Modelling a flood wave due to failure of flood protection measures. DFG-Abschlussbericht KO 1573/15-2, 2008. 
Sebastian Roger, Benjamin Dewals and Holger Schüttrumpf

\section{Hybrid Modelling of Dike-Break Induced Flows}

Experimental model data and numerical computations of dike-break induced flows complement one another within a hybrid approach. Characteristic flow field quantities are recorded with sophisticated measurement techniques during extensive model tests taking into account the specific boundary conditions of a dike break. The corresponding numerical computations are performed by two university codes, namely DGFlow and WOLF 2D, solving the two-dimensional depth-averaged shallow water equations as well as a commercial code STAR-CD based on the Reynolds-averaged Navier-Stokesequations.

\section{Себастьян Рогер, Беньямин Девальс и Хольгер Шюттрумпф}

\section{Гибридное моделирование индуцированных прорывом дамбы течений}

Экспериментальные модельные испытания и цифровое моделирование взаимно дополняют друг друга в гибридной программе исследования индуцированных прорывом дамбы течений. В ходе лабораторных исследований характерные величины поля течения подробно протоколируются с учетом специфических при прорыве дамбы рамочных условий с помощью высокоточной измерительной техники. Соответствующее цифровое моделирование проводится на основе двухмерных, осредненных в отношении глубины уравнений для мелководья, а также уравнений Новье-Стокса, осредненных по Рейнольдсу. 